\title{
SIMPLE MIXING ACTIONS WITH UNCOUNTABLY MANY PRIME FACTORS
}

\author{
ALEXANDRE I. DANILENKO AND ANTON V. SOLOMKO
}

\begin{abstract}
Via $(C, F)$-construction we produce a 2 -fold simple mixing transformation which has uncountably many non-trivial proper factors and all of them are prime.
\end{abstract}

\section{INTRODUCTION}

This paper is about prime factors of simple probability preserving actions. We first recall these and related definitions from the theory of joinings.

Let $T=\left(T_{g}\right)_{g \in \Gamma}$ be an ergodic action of a locally compact second countable group $\Gamma$ on a standard probability space $(X, \mathfrak{B}, \mu)$. The main interest for us lies in $\mathbb{Z}$ and $\mathbb{R}$-actions. A measure $\lambda$ on $X \times X$ is called a 2 -fold self-joining of $T$ if it is

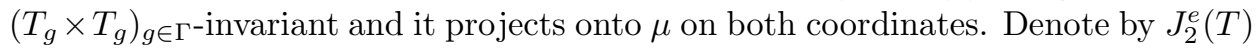
the set of all ergodic 2-fold self-joinings of $T$. Let $C(T)$ stand for the centralizer of $T$, i.e. the set of all $\mu$-preserving invertible transformations of $X$ commuting with $T_{g}$ for each $g \in \Gamma$. Given a transformation $S \in C(T)$, we denote by $\mu_{S}$ the corresponding off-diagonal measure on $X \times X$ defined by $\mu_{S}(A \times B):=\mu\left(A \cap S^{-1} B\right)$ for all $A, B \in \mathfrak{B}$. In other words, $\mu_{S}$ is the image of the measure $\mu$ under the map $x \mapsto(x, S x)$. Of course, $\mu_{S} \in J_{2}^{e}(T)$ for every $S \in C(T)$. If $T$ is weakly mixing, $\mu \times \mu$ is also an ergodic self-joining. If $J_{2}^{e}(T) \subset\left\{\mu_{S} \mid S \in C(T)\right\} \cup\{\mu \times \mu\}$ then $T$ is called 2-fold simple [Ve, dJR. By a factor of $T$ we mean a non-trivial proper $T$-invariant sub- $\sigma$-algebra of $\mathfrak{B}$. If $T$ has no non-trivial proper factors then $T$ is called prime. In $\mathrm{Ve}$ it was shown that if $T$ is 2 -fold simple then for each non-trivial factor $\mathfrak{F}$ of $T$ there exists a compact (in the strong operator topology) subgroup $K_{\mathfrak{F}} \subset C(T)$ such that $\mathfrak{F}=\mathfrak{F}_{K_{\mathfrak{F}}}$, where

$$
\mathfrak{F}_{K}=\{A \in \mathfrak{B} \mid \mu(k A \triangle A)=0 \text { for all } k \in K\}
$$

is the fixed algebra of $K$. In particular, $\mathfrak{F}$ (or, more precisely, the restriction of $T$ to $\mathfrak{F})$ is prime if and only if $K_{\mathfrak{F}}$ is a maximal compact subgroup of $C(T)$.

One of the natural questions arising after the general theory of simple actions was developed in $\mathrm{dJR}$ is: are there simple maps with non-unique prime factors? The first example of such maps was constructed by Glasner and Weiss [GlW] as an inverse limit of certain horocycle flows. For that they used some subtle facts from Ratner's theory of joinings for horocycle flows and properties of lattices in $S L_{2}(\mathbb{R})$. The authors of a later paper [DdJ] utilized a more elementary cutting-and-stacking technique to construct a weakly mixing 2 -fold simple transformation which has countably many factors, all of which are prime. Our purpose in the present paper is to use a similar cutting-and-stacking technique to produce a mixing transformation which has uncountably many factors, all of which are prime.

2010 Mathematics Subject Classification. 37A05, 37A10. 
Via $(C, F)$-construction we produce a measure preserving action $T$ of an auxiliary group $G=\mathbb{Z} \times\left(\mathbb{R} \rtimes \mathbb{Z}_{2}\right)$ such that the transformation $T_{(1,0,0)}$ is mixing 2-fold simple and $C\left(T_{(1,0,0)}\right)=\left\{T_{g} \mid g \in G\right\}$. Since all non-trivial compact subgroups of $G$ are $G_{b}=\{(0,0,0),(0, b, 1)\}, 0 \neq b \in \mathbb{R}$, and all of them are maximal, this gives an example of 2 -fold simple transformation with uncountably many prime factors. All these factors are 2-to-1 and pairwise isomorphic.

We also correct a gap in the proof of [DdJ, Lemma 2.3(ii)] (see Remark 2.4).

The skeleton of the proof of the main result is basically the same as in DdJ, where the "discrete case" (i.e. the auxiliary group is discrete) was under consideration. To work with the $(C, F)$-construction for actions of continuous (i.e. nondiscrete) groups we use the approximation techniques from [Da2].

\section{1. $(C, F)$-CONSTRUCTION}

We now briefly outline the $(C, F)$-construction of measure preserving actions for locally compact groups. For details see [Da1 and references therein.

Let $G$ be a unimodular locally compact second countable (l.c.s.c.) amenable group. Fix a ( $\sigma$-finite) Haar measure $\lambda$ on it. Given two subsets $E, F \subset G$, by $E F$ we mean their algebraic product, i.e. $E F=\{e f \mid e \in E, f \in F\}$. The set $\left\{e^{-1} \mid e \in E\right\}$ is denoted by $E^{-1}$. If $E$ is a singleton, say $E=\{e\}$, then we will write $e F$ for $E F$. Given a finite set $A,|A|$ will denote the cardinality of $A$. Given a subset $F \subset G$ of finite Haar measure, $\lambda_{F}$ will denote the probability on $F$ given by $\lambda_{F}(A):=\lambda(A) / \lambda(F)$ for each measurable $A \subset F$. If $D$ is finite, then $\kappa_{D}$ will denote the equidistributed probability on $D$, that is $\kappa_{D}(A):=|A| /|D|$ for each $A \subset D$.

To define a $(C, F)$-action of $G$ we need two sequences $\left(F_{n}\right)_{n=0}^{\infty}$ and $\left(C_{n}\right)_{n=1}^{\infty}$ of subsets in $G$ such that the following are satisfied:

$$
\begin{aligned}
& \left(F_{n}\right)_{n=0}^{\infty} \text { is a Følner sequence in } G, \\
& C_{n} \text { is finite and }\left|C_{n}\right|>1, \\
& F_{n} C_{n+1} \subset F_{n+1}, \\
& F_{n} c \cap F_{n} c^{\prime}=\varnothing \text { for all } c \neq c^{\prime} \in C_{n+1} .
\end{aligned}
$$

This means that $F_{n} C_{n+1}$ consists of $\left|C_{n+1}\right|$ mutually disjoint 'copies' $F_{n} c, c \in C_{n+1}$, of $F_{n}$ and all these copies are contained in $F_{n+1}$.

First, we define a probability space $(X, \mu)$ in the following way. We equip $F_{n}$ with the measure $\left(\left|C_{1}\right| \cdots\left|C_{n}\right|\right)^{-1} \lambda \uparrow F_{n}$ and endow $C_{n}$ with the equidistributed probability measure. Let $X_{n}:=F_{n} \times \prod_{k>n} C_{k}$ stand for the product of measure spaces. Define an embedding $X_{n} \rightarrow X_{n+1}$ by setting

$$
\left(f_{n}, c_{n+1}, c_{n+2}, \ldots\right) \mapsto\left(f_{n} c_{n+1}, c_{n+2}, \ldots\right) .
$$

It is easy to see that this embedding is measure preserving. Then $X_{1} \subset X_{2} \subset \cdots$. Let $X:=\bigcup_{n=0}^{\infty} X_{n}$ denote the inductive limit of the sequence of measure spaces $X_{n}$ and let $\mathfrak{B}$ and $\mu$ denote the corresponding Borel $\sigma$-algebra and measure on $X$ respectively. Then $X$ is a standard Borel space and $\mu$ is $\sigma$-finite. It is easy to check that $\mu$ is finite if and only if

$$
\lim _{n \rightarrow \infty} \frac{\lambda\left(F_{n}\right)}{\left|C_{1}\right| \cdots\left|C_{n}\right|}<\infty .
$$

If (1.5) is satisfied then we choose (i.e., normalize) $\lambda$ in such a way that $\mu(X)=1$. 
Now we define a $\mu$-preserving action of $G$ on $X$. Suppose that the following is satisfied:

$$
\text { for any } g \in G \text {, there is } m \geqslant 0 \text { with } g F_{n} C_{n+1} \subset F_{n+1} \text { for all } n \geqslant m \text {. }
$$

For such $n$, take $x \in X_{n} \subset X$ and write the expansion $x=\left(f_{n}, c_{n+1}, c_{n+2}, \ldots\right)$ with $f_{n} \in F_{n}$ and $c_{i} \in C_{i}, i>n$. Then we let

$$
T_{g} x:=\left(g f_{n} c_{n+1}, c_{n+2}, \ldots\right) \in X_{n+1} \subset X .
$$

It follows from (1.6) that $T_{g}$ is a well defined $\mu$-preserving transformation of $X$. Moreover, $T_{g} T_{h}=T_{g h}$, i.e. $T:=\left(T_{g}\right)_{g \in G}$ is a $\mu$-preserving Borel action of $G$ on $X$. $T$ is called the $(C, F)$-action of $G$ associated with $\left(C_{n+1}, F_{n}\right)_{n=0}^{\infty}$.

We now recall some basic properties of $(X, \mathfrak{B}, \mu, T)$. Given a Borel subset $A \subset$ $F_{n}$, we put

$$
[A]_{n}:=\left\{x \in X \mid x=\left(f_{n}, c_{n+1}, c_{n+2}, \ldots\right) \in X_{n} \text { and } f_{n} \in A\right\}
$$

and call this set an $n$-cylinder. It is clear that the $\sigma$-algebra $\mathfrak{B}$ is generated by the family of all cylinders. Given Borel subsets $A, B \subset F_{n}$, we have

$$
\begin{aligned}
& {[A \cap B]_{n}=[A]_{n} \cap[B]_{n},[A \cup B]_{n}=[A]_{n} \cup[B]_{n},} \\
& {[A]_{n}=\left[A C_{n+1}\right]_{n+1}=\bigsqcup_{c \in C_{n+1}}[A c]_{n+1},} \\
& \mu\left([A]_{n}\right)=\left|C_{n+1}\right| \mu\left([A c]_{n+1}\right) \text { for every } c \in C_{n+1}, \\
& \mu\left([A]_{n}\right)=\mu\left(X_{n}\right) \lambda_{F_{n}}(A), \\
& T_{g}[A]_{n}=[g A]_{n} \text { if } g A \subset F_{n}, \\
& T_{g}[A]_{n}=T_{h}\left[h^{-1} g A\right]_{n} \text { if } h^{-1} g A \subset F_{n} .
\end{aligned}
$$

Each $(C, F)$-action is of funny rank one (for the definition see $\mathrm{Fe}$ for the case of $\mathbb{Z}$-actions and $[\mathrm{So}$ ] for the general case) and hence ergodic. It also follows from (1.2) that $T$ is conservative.

\section{MAin RESUlt}

By $\mathbb{Z}_{n}$ we denote a cyclic group of order $n$, i.e. $\mathbb{Z}_{n}=\mathbb{Z} / n \mathbb{Z}=\{0,1, \ldots, n-1\}$. Let $G:=\mathbb{Z} \times\left(\mathbb{R} \rtimes \mathbb{Z}_{2}\right)$ with multiplication law as follows

$$
(x, a, n)(y, b, m):=\left(x+y, a+(-1)^{n} b, n+m\right) .
$$

Then the center $C(G)$ of $G$ is $\mathbb{Z} \times\{0\} \times\{0\}$. Each compact subgroup of $G$ coincides with $G_{b}=\{(0,0,0),(0, b, 1)\}$ for some $b \in \mathbb{R}$. Notice that $G_{b}$ is a maximal compact subgroup of $G$ if $b \neq 0$.

To construct the required $(C, F)$-action of $G$ we will determine a sequence $\left(C_{n+1}, F_{n}\right)_{n=0}^{\infty}$. Let $\left(r_{n}\right)_{n=0}^{\infty}$ be an increasing sequence of positive integers such that

$$
\lim _{n \rightarrow \infty} \frac{n^{4}}{r_{n}}=0
$$

Below - just after Lemma 2.1 — one more restriction on the growth of $\left(r_{n}\right)_{n=0}^{\infty}$ will be imposed and we will assume that $r_{n}$ is large so that (2.6) is satisfied. We define recurrently three other sequences $\left(\widetilde{a}_{n}\right)_{n=0}^{\infty},\left(a_{n}\right)_{n=1}^{\infty}$ and $\left(b_{n}\right)_{n=1}^{\infty}$ of positive integers by setting

$$
\widetilde{a}_{0}:=1
$$




$$
\begin{aligned}
& a_{n}:=\left(2 r_{n-1}+1\right) \widetilde{a}_{n-1} \text { for } n \geqslant 1, \\
& b_{n}:=(2 n-1) \widetilde{a}_{n-1} \text { for } n \geqslant 1, \\
& \widetilde{a}_{n}:=a_{n}+b_{n}+n \text { for } n \geqslant 1 .
\end{aligned}
$$

For each $n \in \mathbb{N}$, we let

$$
\begin{aligned}
I_{n} & :=\{-n, \ldots, n\}^{2} \subset \mathbb{Z}^{2}, \\
H_{n} & :=\left\{-r_{n}, \ldots, r_{n}\right\}^{2} \subset \mathbb{Z}^{2}, \\
F_{n} & :=\left(-a_{n}, a_{n}\right]_{\mathbb{Z}} \times\left(-a_{n}, a_{n}\right]_{\mathbb{R}} \times \mathbb{Z}_{2}, \\
S_{n} & :=\left(-b_{n}, b_{n}\right]_{\mathbb{Z}} \times\left(-b_{n}, b_{n}\right]_{\mathbb{R}} \times \mathbb{Z}_{2}, \\
\widetilde{F}_{n} & :=\left(-\widetilde{a}_{n}, \widetilde{a}_{n}\right]_{\mathbb{Z}} \times\left(-\widetilde{a}_{n}, \widetilde{a}_{n}\right]_{\mathbb{R}} \times \mathbb{Z}_{2} .
\end{aligned}
$$

We also consider a homomorphism $\phi_{n}: \mathbb{Z}^{2} \rightarrow G$ given by

$$
\phi_{n}(i, j):=\left(2 i \widetilde{a}_{n}, 2 j \widetilde{a}_{n}, 0\right) .
$$

We then have

$$
\begin{gathered}
S_{n} \subset F_{n}, \quad F_{n} S_{n}=S_{n} F_{n} \subset \widetilde{F}_{n} \subset G, \\
S_{n+1}=\widetilde{F}_{n} \phi_{n}\left(I_{n}\right)=\bigsqcup_{h \in I_{n}} \widetilde{F}_{n} \phi_{n}(h)=\bigsqcup_{h \in I_{n}} \phi_{n}(h) \widetilde{F}_{n}, \\
F_{n+1}=\widetilde{F}_{n} \phi_{n}\left(H_{n}\right)=\bigsqcup_{h \in H_{n}} \widetilde{F}_{n} \phi_{n}(h)=\bigsqcup_{h \in H_{n}} \phi_{n}(h) \widetilde{F}_{n},
\end{gathered}
$$

Suppose also that $F_{n}$ is equipped with a finite partition $\xi_{n}$ such that the following are satisfied:

(i) the diameter of each atom of $\xi_{n}$ is less than $\frac{1}{n}$,

(ii) for each atom $A \in \xi_{n-1}$ and each element $c \in C_{n}$, the subset $A c \subset F_{n}$ is $\xi_{n}$-measurable and

(iii) $\xi_{n}$ is symmetric, that is $A^{-1} \in \xi_{n}$ whenever $A \in \xi_{n}$.

It follows that for each measurable subset $A \subset F_{n}$, any $\varepsilon>0$ and for all $k$ large enough, there is a $\xi_{k}$-measurable subset $B \subset F_{k}$ such that $\mu\left([A]_{n} \triangle[B]_{k}\right)<\varepsilon$. We will denote by $\sigma\left(\xi_{n}\right)$ the $\sigma$-algebra on $F_{n}$ generated by $\xi_{n}$.

For a finite subset $D$ in $S_{n}$, we denote by $\kappa_{D}$ the corresponding normalized Dirac comb, i.e. a measure on $S_{n}$ given by $\kappa_{D}(A):=\frac{|A \cap D|}{|D|}$ for each subset $A \subset S_{n}$. Given two subsets $A, B \subset F_{n}$ define a function $f_{A, B}: S_{n} \times S_{n} \rightarrow \mathbb{R}$ by setting $f_{A, B}(x, y):=\frac{\lambda(A x \cap B y)}{\lambda\left(F_{n}\right)}, x, y \in S_{n}$. Choose a finite subset $D_{n}$ in $S_{n}$ such that

$$
\left|\int_{S_{n} \times S_{n}} f_{A g, B h} d \kappa_{D_{n}} d \kappa_{D_{n}}-\frac{1}{\lambda\left(S_{n}\right)^{2}} \int_{S_{n} \times S_{n}} f_{A g, B h} d \lambda d \lambda\right|<\frac{1}{n}
$$

for each $\xi_{n}$-measurable subsets $A, B \subset F_{n}$ and any $g, h \in F_{n}$ with $A g S_{n}, B h S_{n} \subset$ $F_{n}$. For instance, let $\xi_{n}$ consists of 'rectangles' $\{a\} \times \Delta \times\{m\} \subset G$, where $a \in$ $\left(-a_{n}, a_{n}\right]_{\mathbb{Z}}, m \in \mathbb{Z}_{2}$ and $\Delta \subset\left(-a_{n}, a_{n}\right]_{\mathbb{R}}$ is a subinterval of length $n^{-1}$, and set $D_{n}:=\left\{\left(a, k n^{-2}, m\right) \mid a \in\left(-b_{n}, b_{n}\right]_{\mathbb{Z}}, k \in\left(-n^{2} b_{n}, n^{2} b_{n}\right]_{\mathbb{Z}}, m \in \mathbb{Z}_{2}\right\}$. It is an easy exercise to check that (2.5) is satisfied for such $\xi_{n}$ and $D_{n}$. We notice also that $\left|D_{n}^{0}\right|=\left|D_{n}^{1}\right|$. 
Given a finite (signed) measure $\nu$ on a finite set $D$, we let $\|\nu\|_{1}:=\sum_{d \in D}|\nu(d)|$. If $\pi: D \rightarrow E$ then clearly $\left\|\nu \circ \pi^{-1}\right\|_{1} \leqslant\|\nu\|_{1}$. Given a finite set $Y$ and a mapping $s: Y \rightarrow D$, let $\operatorname{dist}_{y \in Y} s(y)$ denote the image of the equidistribution on $Y$ under $s$ :

$$
\operatorname{dist}_{y \in Y} s(y):=\frac{1}{|Y|} \sum_{y \in Y} \delta_{s(y)}=\kappa_{D} \circ s^{-1} .
$$

The following lemma easily follows from [dJ, Lemma 2.1] (cf. [Da2, Lemma 3.2]).

Lemma 2.1. Let $D$ be a finite set. Then given $\varepsilon>0$ and $\delta>0$, there is $R \in \mathbb{N}$ such that for each $r>R$, there exists a map $s:\{-r, \ldots, r\}^{2} \rightarrow D$ such that

$$
\| \text { dist }_{0 \leqslant t<N}\left(s_{n}(h+(t, 0)), s_{n}\left(h^{\prime}+(t, 0)\right)\right)-\kappa_{D} \times \kappa_{D} \|_{1}<\varepsilon
$$

for each $N>\delta r$ and $h \neq h^{\prime} \in\{-r, \ldots, r\}^{2}$ with $h_{1}+N<r$ and $h_{1}^{\prime}+N<r$ !

Applying this Lemma with $\varepsilon=\frac{1}{n}$ and $\delta=\frac{1}{n^{2}}$ we get the following. If $r_{n}$ is large enough then there is a mapping $s_{n}: H_{n} \rightarrow D_{n}$ such that for any $N>\frac{r_{n}}{n^{2}}$ and $h \neq h^{\prime} \in H_{n} \cap\left(H_{n}-(N-1,0)\right)$ we have

$$
\left\|\operatorname{dist}_{0 \leqslant t<N}\left(s_{n}(h+(t, 0)), s_{n}\left(h^{\prime}+(t, 0)\right)\right)-\kappa_{D_{n}} \times \kappa_{D_{n}}\right\|_{1}<\frac{1}{n} .
$$

From now on we will assume that $r_{n}$ is large so that this condition is satisfied and for each $n$ fix $s_{n}: H_{n} \rightarrow D_{n}$ satisfying (2.6).

Now we define a map $c_{n+1}: H_{n} \rightarrow G$ by setting $c_{n+1}(h):=s_{n}(h) \phi_{n}(h)$. We put $C_{n+1}:=c_{n+1}\left(H_{n}\right)$.

The reader should have the following picture in mind. The set $F_{n+1}$ is exactly tiled with the sets $\widetilde{F}_{n} \phi_{n}(h), h \in H_{n}$, which may be thought of as 'windows'. Each $F_{n}$ has a 'natural' translate $F_{n} \phi_{n}(h)$ in $\widetilde{F}_{n} \phi_{n}(h)$ but the translate we actually choose is the natural translate perturbed by a further translation $s_{n}(h)$ which is chosen in a 'random' way and does not move $F_{n} \phi_{n}(h)$ out of its window.

It is easy to derive that (1.1)-(1.6) are satisfied for the sequence $\left(F_{n}, C_{n+1}\right)_{n=0}^{\infty}$. Hence the associated $(C, F)$-action $T=\left(T_{g}\right)_{g \in G}$ of $G$ is well defined on a standard probability space $(X, \mathfrak{B}, \mu)$.

We now state the main result.

Theorem 2.2. The transformation $T_{(1,0,0)}$ is mixing and 2-fold simple. All nontrivial proper factors of $T_{(1,0,0)}$ are of the form $\mathfrak{F}_{G_{b}}, 0 \neq b \in \mathbb{R}$. All these factors are 2-to-1, prime and pairwise isomorphic.

We first prove some technical lemmata. After that in Proposition 2.8 we show mixing for $T_{(1,0,0)}$ and in Proposition 2.9 we prove simplicity and describe the centralizer of $T_{(1,0,0)}$. The structure of factors follows then from Veech's theorem.

Denote by $G^{0}$ the subgroup $\mathbb{Z} \times \mathbb{R} \times\{0\}$ of index 2 in $G$. Given any subset $A$ in $G$ we set $A^{0}:=A \cap G^{0}$ and $A^{1}:=A \backslash A^{0}$. We will refer to $A^{0}$ and $A^{1}$ as 'levels' of $A$. We will say that a subset $A \subset G$ is $\varepsilon$-balanced if

$$
\left|\lambda\left(A^{0}\right)-\lambda\left(A^{1}\right)\right|<\varepsilon \lambda(A) .
$$

Denote by $\pi_{3}: G \rightarrow \mathbb{Z}_{2}$ a natural projection on the third coordinate. Since $\kappa_{D_{n}} \circ$ $\pi_{3}^{-1}=\kappa_{\mathbb{Z}_{2}}$, it follows from (2.6) that

$$
\left\|\operatorname{dist}_{h \in H_{n}} \pi_{3} \circ s_{n}(h)-\kappa_{\mathbb{Z}_{2}}\right\|_{1}<\frac{1}{n} .
$$

\footnotetext{
${ }^{1}$ Here and below by $a \neq b \in A$ we denote two different elements $a, b$ of a set $A$.
} 
In particular, for any $A^{*} \subset F_{n}$ the set $A=A^{*} C_{n+1}$ is $\frac{1}{n}$-balanced:

$$
\left|\lambda\left(A^{0}\right)-\lambda\left(A^{1}\right)\right|<\frac{1}{n} \lambda(A) .
$$

Indeed, since

$$
\begin{aligned}
& A^{0}=\bigsqcup_{h \in s_{n}^{-1}\left(G^{0}\right)} A^{* 0} c_{n}(h) \sqcup \bigsqcup_{h \in s_{n}^{-1}\left(G^{1}\right)} A^{* 1} c_{n}(h), \\
& \lambda\left(A^{0}\right)=\lambda\left(A^{* 0}\right)\left|s_{n}^{-1}\left(G^{0}\right)\right|+\lambda\left(A^{* 1}\right)\left|s_{n}^{-1}\left(G^{1}\right)\right|,
\end{aligned}
$$

and similarly

$$
\lambda\left(A^{1}\right)=\lambda\left(A^{* 1}\right)\left|s_{n}^{-1}\left(G^{0}\right)\right|+\lambda\left(A^{* 0}\right)\left|s_{n}^{-1}\left(G^{1}\right)\right| .
$$

Hence

$$
\begin{aligned}
\left|\lambda\left(A^{0}\right)-\lambda\left(A^{1}\right)\right| & =\left|\lambda\left(A^{* 0}\right)-\lambda\left(A^{* 1}\right)\right||| s_{n}^{-1}\left(G^{0}\right)|-| s_{n}^{-1}\left(G^{1}\right)|| \\
& \leqslant \frac{1}{\left|H_{n}\right|} \lambda(A)|| s_{n}^{-1}\left(G^{0}\right)|-| s_{n}^{-1}\left(G^{1}\right)|| .
\end{aligned}
$$

It remains to notice that

$$
\begin{aligned}
\frac{1}{\left|H_{n}\right|}|| s_{n}^{-1}\left(G^{0}\right)|-| s_{n}^{-1}\left(G^{1}\right)|| & \leqslant\left|\frac{\left|s_{n}^{-1}\left(G^{0}\right)\right|}{\left|H_{n}\right|}-\frac{1}{2}\right|+\left|\frac{\left|s_{n}^{-1}\left(G^{1}\right)\right|}{\left|H_{n}\right|}-\frac{1}{2}\right| \\
& =\left\|\operatorname{dist}_{h \in H_{n}} \pi_{3} \circ s_{n}(h)-\kappa_{\mathbb{Z}_{2}}\right\|_{1}<\frac{1}{n}
\end{aligned}
$$

by (2.8). It follows that $A=A^{*} C_{n+1}$ is $\frac{1}{n}$-balanced for each $A^{*} \subset F_{n}$.

Given $h=\left(h_{1}, h_{2}\right) \in \mathbb{Z}^{2}$, we let $h^{*}:=\left(h_{1},-h_{2}\right)$.

Lemma 2.3. Let $f=f^{\prime} \phi_{n-1}(h)$ with $f^{\prime} \in \widetilde{F}_{n-1}$ and $h \in \mathbb{Z}^{2}$.

(i) Suppose $f \in G^{\alpha}$ and let $\beta:=1-\alpha$. Let

$$
\begin{aligned}
& L_{n}^{-}:=\widetilde{F}_{n-1}^{\alpha} \phi_{n-1}\left(I_{n-2}+h\right) \sqcup \widetilde{F}_{n-1}^{\beta} \phi_{n-1}\left(I_{n-2}+h^{*}\right) \text { and } \\
& L_{n}^{+}:=\widetilde{F}_{n-1}^{\alpha} \phi_{n-1}\left(I_{n}+h\right) \sqcup \widetilde{F}_{n-1}^{\beta} \phi_{n-1}\left(I_{n}+h^{*}\right) .
\end{aligned}
$$

Then $L_{n}^{-} \subset f S_{n} \subset L_{n}^{+}$. Hence

$$
\frac{\lambda\left(f S_{n} \triangle L_{n}^{-}\right)}{\lambda\left(S_{n}\right)}=\bar{o}(1)
$$

(ii) If, in addition, $f S_{n} \subset F_{n}$ then for any subset $A=A^{*} C_{n-1}$ with $A^{*} \subset F_{n-2}$ we have

$$
\frac{\lambda\left(A C_{n} \cap f S_{n}\right)}{\lambda\left(S_{n}\right)}=\lambda_{F_{n-1}}(A)+\bar{o}(1) .
$$

Here $\bar{o}(1)$ means a sequence that goes to 0 as $n \rightarrow \infty$ and does not depend on the choice of $A^{*}$ in $F_{n-2}$.

Proof. (i) Suppose $f \in G^{0}$ (the case $f \in G^{1}$ is considered in a similar way). We have

$$
\begin{aligned}
f S_{n} & =f^{\prime} \phi_{n-1}(h) \widetilde{F}_{n-1} \phi_{n-1}\left(I_{n-1}\right) \\
& =f^{\prime} \widetilde{F}_{n-1}^{0} \phi_{n-1}\left(h+I_{n-1}\right) \sqcup f^{\prime} \widetilde{F}_{n-1}^{1} \phi_{n-1}\left(h^{*}+I_{n-1}\right) .
\end{aligned}
$$


Since $\widetilde{F}_{n-1}^{0} \widetilde{F}_{n-1}^{\alpha} \subset \bigsqcup_{u \in I_{1}} \widetilde{F}_{n-1}^{\alpha} \phi_{n-1}(u)$, there exists a partition of $\widetilde{F}_{n-1}^{\alpha}$ into subsets $A_{u}^{\alpha}, u \in I_{1}$, such that $f^{\prime} A_{u}^{\alpha} \subset \widetilde{F}_{n-1}^{\alpha} \phi_{n-1}(u)$ for any $u$ and $\alpha=0,1$. Therefore

$f S_{n}=\bigsqcup_{u \in I_{1}}\left(f^{\prime} A_{u}^{0} \phi_{n-1}(u)^{-1} \phi_{n-1}\left(u+h+I_{n-1}\right) \sqcup f^{\prime} A_{u}^{1} \phi_{n-1}(u)^{-1} \phi_{n-1}\left(u+h^{*}+I_{n-1}\right)\right)$.

It remains to notice that $\bigsqcup_{u \in I_{1}} f^{\prime} A_{u}^{\alpha} \phi_{n-1}(u)^{-1}=\widetilde{F}_{n-1}^{\alpha}$.

(ii) Since $f S_{n} \subset F_{n}$ and $F_{n}=\widetilde{F}_{n-1} \phi_{n-1}\left(H_{n-1}\right)$, it follows from (i) that the subsets $K:=I_{n-1}+h$ and $K^{*}:=I_{n-1}+h^{*}$ are contained in $H_{n-1}$. Therefore

$$
\begin{aligned}
& \frac{\lambda\left(A C_{n} \cap f S_{n}\right)}{\lambda\left(S_{n}\right)}=\sum_{k \in H_{n-1}} \frac{\lambda\left(A c_{n}(k) \cap f S_{n}\right)}{\lambda\left(S_{n}\right)} \\
& =\sum_{k \in H_{n-1}} \frac{\lambda\left(A c_{n}(k) \cap L_{n}^{-}\right)}{\lambda\left(S_{n}\right)}+\bar{o}(1) \\
& =\frac{1}{\lambda\left(S_{n}\right)} \sum_{k \in H_{n-1}} \lambda\left(A s_{n-1}(k) \phi_{n-1}(k) \cap \widetilde{F}_{n-1}^{\alpha} \phi_{n-1}(K) \sqcup \widetilde{F}_{n-1}^{\beta} \phi_{n-1}\left(K^{*}\right)\right)+\bar{o}(1) \\
& =\frac{1}{\lambda\left(S_{n}\right)} \sum_{k \in K} \lambda\left(A s_{n-1}(k) \cap \widetilde{F}_{n-1}^{\alpha}\right)+\frac{1}{\lambda\left(S_{n}\right)} \sum_{k \in K^{*}} \lambda\left(A s_{n-1}(k) \cap \widetilde{F}_{n-1}^{\beta}\right)+\bar{o}(1) .
\end{aligned}
$$

Notice that

$$
\lambda\left(A s_{n-1}(k) \cap \widetilde{F}_{n-1}^{\alpha}\right)=\left\{\begin{array}{l}
\lambda\left(A^{\alpha}\right), \text { if } s_{n-1}(k) \in G^{0} \\
\lambda\left(A^{\beta}\right), \text { if } s_{n-1}(k) \in G^{1} .
\end{array}\right.
$$

In any case, since $A=A^{\prime} C_{n-1}$ is $\frac{1}{n-2}$-balanced, we conclude from (2.8) that

$$
\lambda\left(A s_{n-1}(k) \cap \widetilde{F}_{n-1}^{\alpha}\right)=\left(\frac{1}{2}+\bar{o}(1)\right) \lambda(A) .
$$

In a similar way

$$
\lambda\left(A s_{n-1}(k) \cap \widetilde{F}_{n-1}^{\beta}\right)=\left(\frac{1}{2}+\bar{o}(1)\right) \lambda(A) .
$$

Hence

$$
\begin{aligned}
& \frac{\lambda\left(A C_{n} \cap f S_{n}\right)}{\lambda\left(S_{n}\right)}= \frac{\lambda(A)|K|(1+\bar{o}(1))}{\lambda\left(S_{n}\right)}+\bar{o}(1) \\
&=\frac{\lambda(A)}{\lambda\left(F_{n-1}\right)} \cdot \frac{\lambda\left(F_{n-1}\right)|K|}{\lambda\left(S_{n}\right)} \cdot(1+\bar{o}(1))+\bar{o}(1) \\
&=\lambda_{F_{n-1}}(A) \cdot \frac{\lambda\left(F_{n-1}\right)(2 n-1)^{2}}{(2 n+1)^{2} \lambda\left(\widetilde{F}_{n-1}\right)} \cdot(1+\bar{o}(1))+\bar{o}(1)=\lambda_{F_{n-1}}(A)+\bar{o}(1) .
\end{aligned}
$$

Remark 2.4. We note that there is a gap in [DdJ, Lemma 2.3(ii)]. It was stated there that the claim (ii) is true for each subset $A \subset F_{n-1}$. This is not true. However - as was shown in Lemma 2.3(ii) above - the claim is true if $A=A^{*} C_{n-1}$ for an arbitrary subset $A^{*} \subset F_{n-2}$. This corrected version of the claim suffices to apply it in the proof of [DdJ, Theorem 2.5] which is the only place in that paper where DdJ, Lemma 2.3(ii)] was used.

We will also use the following simple lemma. 
Lemma 2.5. Let $A, B$ and $S$ be subsets of finite Haar measure in $G$. Then

$$
\int_{S \times S} \lambda(A x \cap B y) d \lambda(x) d \lambda(y)=\int_{A \times B} \lambda(a S \cap b S) d \lambda(a) d \lambda(b) .
$$

Proof. Notice that $G$ is unimodular. Consider two subsets in $G^{3}$ :

$$
\begin{aligned}
\Omega_{1}: & =\left\{(a, x, y) \mid x \in S, y \in S, a \in A \cap B y x^{-1}\right\} \\
& =\left\{(a, x, y) \mid a \in A, y \in S, x \in a^{-1} B y \cap S\right\} \text { and } \\
\Omega_{2}: & =\left\{(a, b, y) \mid a \in A, b \in B, y \in b^{-1} a S \cap S\right\} \\
& =\left\{(a, b, y) \mid a \in A, y \in S, b \in B \cap a S y^{-1}\right\} .
\end{aligned}
$$

It is clear that the maping $\Omega_{1} \ni(a, x, y) \mapsto\left(a, a x y^{-1}, y\right) \in \Omega_{2}$ is 1-to- 1 and $\lambda^{3}$ preserving. Applying Fubini theorem we obtain that

$$
\int_{S \times S} \lambda(A x \cap B y) d \lambda(x) d \lambda(y)=\lambda^{3}\left(\Omega_{1}\right)=\lambda^{3}\left(\Omega_{2}\right)=\int_{A \times B} \lambda(a S \cap b S) d \lambda(a) d \lambda(b) .
$$

The following lemma is the first step to prove mixing for $T_{(1,0,0)}$. Let $h_{0}:=$ $(1,0) \in \mathbb{Z}^{2}$. Then $\phi_{n}\left(h_{0}\right)=(1,0,0)^{2 \widetilde{a}_{n}}$.

Lemma 2.6. Given a sequence of subsets $H_{n}^{*} \subset H_{n}$ such that $\frac{\left|H_{n}^{*}\right|}{\left|H_{n}\right|} \rightarrow \delta$ for some $\delta \geqslant 0$, we let $C_{n}^{*}:=c_{n}\left(H_{n-1}^{*}\right)$. Then

$$
\sup _{A^{*}, B^{*} \in \sigma\left(\xi_{n-1}\right)}\left|\mu\left(T_{\phi_{n}\left(h_{0}\right)}\left[A^{*} C_{n}^{*}\right]_{n} \cap\left[B^{*}\right]_{n-1}\right)-\mu\left(\left[A^{*} C_{n}^{*}\right]_{n}\right) \mu\left(\left[B^{*}\right]_{n-1}\right)\right| \rightarrow 0 .
$$

Proof. Let $A, B \subset F_{n}$ be $\xi_{n}$-measurable. We set $F_{n}^{\circ}:=\left\{f \in F_{n} \mid f S_{n} S_{n}^{-1} \subset F_{n}\right\}$, $A^{\circ}:=A \cap F_{n}^{\circ}, B^{\circ}:=B \cap F_{n}^{\circ}, H_{n}^{\prime}:=H_{n} \cap\left(H_{n}-h_{0}\right)$. It is clear that $\mu\left(F_{n} \backslash F_{n}^{\circ}\right) \rightarrow 0$ and $\frac{\left|H_{n}^{\prime}\right|}{\left|H_{n}\right|} \rightarrow 1$ as $n \rightarrow \infty$. Since $\phi_{n}\left(h_{0}\right) \in C(G)$ for all $n \in \mathbb{N}$, we have

$$
\phi_{n}\left(h_{0}\right) A c_{n+1}(h)=A s_{n}(h) \phi_{n}\left(h_{0}+h\right)=A s_{n}(h) s_{n}\left(h_{0}+h\right)^{-1} c_{n+1}\left(h_{0}+h\right) .
$$

whenever $h \in H_{n}^{\prime}$. In particular, $\phi_{n}\left(h_{0}\right) A^{\circ} c_{n+1}(h) \subset F_{n+1}$ for all $h \in H_{n}^{\prime}$. Then

$$
\begin{aligned}
\left.\left.\mu\left(T_{\phi_{n}\left(h_{0}\right)}\right] A\right]_{n} \cap[B]_{n}\right)=\mu\left(T_{\phi_{n}\left(h_{0}\right)}\left[A^{\circ}\right]_{n} \cap\left[B^{\circ}\right]_{n}\right)+\bar{o}(1) \\
=\sum_{h \in H_{n}} \mu\left(T_{\phi_{n}\left(h_{0}\right)}\left[A^{\circ} c_{n+1}(h)\right]_{n+1} \cap\left[B^{\circ}\right]_{n}\right)+\bar{o}(1) \\
=\sum_{h \in H_{n}^{\prime}} \mu\left(T_{\phi_{n}\left(h_{0}\right)}\left[A^{\circ} c_{n+1}(h)\right]_{n+1} \cap\left[B^{\circ}\right]_{n}\right)+\bar{o}(1) \\
=\sum_{h \in H_{n}^{\prime}} \mu\left(\left[A^{\circ} s_{n}(h) s_{n}\left(h_{0}+h\right)^{-1} c_{n+1}\left(h_{0}+h\right)\right]_{n+1} \cap\left[B^{\circ}\right]_{n}\right)+\bar{o}(1) \\
=\sum_{h \in H_{n}^{\prime}} \mu\left(\left[\left(A^{\circ} s_{n}(h) s_{n}\left(h_{0}+h\right)^{-1} \cap B^{\circ}\right) c_{n+1}\left(h_{0}+h\right)\right]_{n+1}\right)+\bar{o}(1) \\
=\frac{1}{\left|H_{n}\right|} \sum_{h \in H_{n}^{\prime}} \mu\left(\left[A^{\circ} s_{n}(h) s_{n}\left(h_{0}+h\right)^{-1} \cap B^{\circ}\right]_{n}\right)+\bar{o}(1) \\
=\frac{1}{\left|H_{n}\right|} \sum_{h \in H_{n}^{\prime}} \lambda_{F_{n}}\left(A^{\circ} s_{n}(h) \cap B^{\circ} s_{n}\left(h_{0}+h\right)\right) \mu\left(X_{n}\right)+\bar{o}(1)
\end{aligned}
$$




$$
\begin{aligned}
& =\frac{1}{\left|H_{n}^{\prime}\right|} \sum_{h \in H_{n}^{\prime}} \lambda_{F_{n}}\left(A^{\circ} s_{n}(h) \cap B^{\circ} s_{n}\left(h_{0}+h\right)\right)+\bar{o}(1) \\
& =\frac{1}{\left|H_{n}^{\prime}\right|} \sum_{h \in H_{n}^{\prime}} \lambda_{F_{n}}\left(A s_{n}(h) \cap B s_{n}\left(h_{0}+h\right)\right)+\bar{o}(1) .
\end{aligned}
$$

Let $\nu_{n}:=\operatorname{dist}_{h \in H_{n}^{\prime}}\left(s_{n}(h), s_{n}\left(h+h_{0}\right)\right)$. Set $f_{A, B}(x, y):=\lambda_{F_{n}}(A x \cap B y)=\frac{\lambda(A x \cap B y)}{\lambda\left(F_{n}\right)}$. Notice that

$$
\nu_{n}=\frac{1}{2 r_{n}-1} \sum_{i=-r_{n}}^{r_{n}} \operatorname{dist}_{-r_{n} \leqslant t<r_{n}}\left(s_{n}(t, i), s_{n}(t+1, i)\right) .
$$

It follows from (2.6) that $\left\|\nu_{n}-\kappa_{D_{n}} \times \kappa_{D_{n}}\right\|_{1}<\frac{1}{n}$. Then by (2.5)

$$
\begin{aligned}
& \mu\left(T_{\phi_{n}\left(h_{0}\right)}[A]_{n} \cap[B]_{n}\right)=\int_{S_{n} \times S_{n}} f_{A, B} d \nu_{n}+\bar{o}(1) \\
&=\int_{S_{n} \times S_{n}} f_{A, B} d \kappa_{D_{n}} d \kappa_{D_{n}}+\bar{o}(1)=\frac{1}{\lambda\left(S_{n}\right)^{2}} \int_{S_{n} \times S_{n}} f_{A, B} d \lambda d \lambda+\bar{o}(1),
\end{aligned}
$$

Now take $A:=A^{*} C_{n}^{*}$ and $B:=B^{*} C_{n}$ for some $\xi_{n-1}$-measurable subsets $A^{*}, B^{*} \subset$ $F_{n-1}$. We say that elements $c$ and $c^{\prime}$ of $C_{n}$ are partners if $F_{n-1} c S_{n} \cap F_{n-1} c^{\prime} S_{n} \neq \varnothing$. We then write $c \bowtie c^{\prime}$. Since $A^{*} c x \cap B^{*} c^{\prime} y=\varnothing$ for $c \bowtie c^{\prime}$, it follows that

$$
\begin{aligned}
\int_{S_{n} \times S_{n}} f_{A, B} d \lambda d \lambda & =\int_{S_{n} \times S_{n}} \lambda_{F_{n}}\left(A^{*} C_{n} x \cap B^{*} C_{n} y\right) d \lambda(x) d \lambda(y) \\
& =\frac{1}{\lambda\left(F_{n}\right)} \int_{S_{n} \times S_{n}} \sum_{C_{n}^{*} \ni c \bowtie c^{\prime} \in C_{n}} \lambda\left(A^{*} c x \cap B^{*} c^{\prime} y\right) d \lambda(x) d \lambda(y) .
\end{aligned}
$$

Applying Lemma 2.5 we now obtain that

$$
\int_{S_{n} \times S_{n}} f_{A, B} d \lambda d \lambda=\frac{1}{\lambda\left(F_{n}\right)} \sum_{C_{n}^{*} \ni c \bowtie c^{\prime} \in C_{n}} \int_{A^{*} \times B^{*}} \lambda\left(a c S_{n} \cap b c^{\prime} S_{n}\right) d \lambda(a) d \lambda(b) .
$$

Next, we note that

$$
\left|\lambda\left(a c S_{n} \cap b c^{\prime} S_{n}\right)-\lambda\left(c S_{n} \cap c^{\prime} S_{n}\right)\right| \leqslant 8 n \lambda\left(\widetilde{F}_{n-1}\right)=\bar{o}(1) \lambda\left(S_{n}\right) .
$$

Each $c \in C_{n}$ has no more than $2(4 n+1)^{2}$ partners. Therefore

$$
\begin{aligned}
& \mu\left(T_{\phi_{n}\left(h_{0}\right)}\left[A^{*} C_{n}^{*}\right]_{n} \cap\left[B^{*}\right]_{n-1}\right) \\
& =\frac{1}{\lambda\left(S_{n}\right)^{2}} \sum_{C_{n}^{*} \ni c \bowtie c^{\prime} \in C_{n}} \int_{A^{*} \times B^{*}} \frac{\lambda\left(c S_{n} \cap c^{\prime} S_{n}\right)+\lambda\left(S_{n}\right) \bar{o}(1)}{\lambda\left(F_{n}\right)} d \lambda(a) d \lambda(b)+\bar{o}(1) \\
& =\frac{\lambda\left(A^{*}\right) \lambda\left(B^{*}\right)}{\lambda\left(F_{n-1}\right)^{2}} \frac{\lambda\left(F_{n-1}\right)^{2}}{\lambda\left(S_{n}\right)^{2} \lambda\left(F_{n}\right)} \sum_{C_{n}^{*} \ni c \bowtie c^{\prime} \in C_{n}}\left(\lambda\left(c S_{n} \cap c^{\prime} S_{n}\right)+\lambda\left(S_{n}\right) \bar{o}(1)\right)+\bar{o}(1) \\
& =\lambda_{F_{n-1}}\left(A^{*}\right) \lambda_{F_{n-1}}\left(B^{*}\right) \theta_{n} \pm \frac{\lambda\left(F_{n-1}\right)^{2}\left|H_{n}^{*}\right| 2(4 n+1)^{2} \lambda\left(S_{n}\right) \bar{o}(1)}{\lambda\left(S_{n}\right)^{2} \lambda\left(F_{n}\right)}+\bar{o}(1) \\
& =\lambda_{F_{n-1}}\left(A^{*}\right) \lambda_{F_{n-1}}\left(B^{*}\right) \theta_{n} \pm \frac{\lambda\left(F_{n-1}\right)^{2}\left|H_{n}^{*}\right| 2(4 n+1)^{2} \bar{o}(1)}{\lambda\left(\widetilde{F}_{n-1}\right)^{2}(2 n-1)^{2}\left|H_{n}\right|}+\bar{o}(1) \\
& =\lambda_{F_{n-1}}\left(A^{*}\right) \lambda_{F_{n-1}}\left(B^{*}\right) \theta_{n}+\bar{o}(1),
\end{aligned}
$$


where $\theta_{n}=\frac{\lambda\left(F_{n-1}\right)^{2}}{\lambda\left(S_{n}\right)^{2} \lambda\left(F_{n}\right)} \sum_{C_{n}^{*} \ni c \bowtie c^{\prime} \in C_{n}} \lambda\left(c S_{n} \cap c^{\prime} S_{n}\right)$. Substituting $A^{*}=B^{*}=F_{n-1}$ and passing to the limit we obtain that $\theta_{n} \rightarrow \delta$ as $n \rightarrow \infty$. Hence

$$
\mu\left(T_{\phi_{n}\left(h_{0}\right)}\left[A^{*} C_{n}^{*}\right]_{n} \cap\left[B^{*}\right]_{n-1}\right)=\mu\left(\left[A^{*} C_{n}^{*}\right]_{n}\right) \mu\left(\left[B^{*}\right]_{n-1}\right)+\bar{o}(1) .
$$

Since $\bar{o}(1)$ does not depend on the choice of $A^{*}$ and $B^{*}$ inside $F_{n-1}$, the claim is proven.

Corollary 2.7. The transformation $T_{(1,0,0)}$ is weakly mixing.

Proof. Substituting $H_{n}^{*}:=H_{n}$ to (2.9) we obtain that

$$
\sup _{A^{*}, B^{*} \in \sigma\left(\xi_{n-1}\right)}\left|\mu\left(T_{\phi_{n}\left(h_{0}\right)}\left[A^{*}\right]_{n-1} \cap\left[B^{*}\right]_{n-1}\right)-\mu\left(\left[A^{*}\right]_{n-1}\right) \mu\left(\left[B^{*}\right]_{n-1}\right)\right| \rightarrow 0 .
$$

Since each measurable subset of $X$ can be approximated by $\left[A^{*}\right]_{n-1}$ for large $n$ and $\xi_{n-1}$-measurable subset $A^{*} \subset F_{n-1}$, it follows that the sequence $\left(\phi_{n}\left(h_{0}\right)\right)_{n=1}^{\infty}$ is mixing for $T$, that is $\mu\left(T_{\phi_{n}\left(h_{0}\right)} A \cap B\right) \rightarrow \mu(A) \mu(B)$ for every pair of measurable subsets $A, B \subset X$.

Proposition 2.8. The transformation $T_{(1,0,0)}$ is mixing.

Proof. We have to show that

$$
\lim _{n \rightarrow \infty} \mu\left(T_{g_{n}} A \cap B\right)=\mu(A) \mu(B)
$$

for any sequence $\left(g_{n}\right)_{n=1}^{\infty}$ that goes to infinity in $C(G)$ and every pair of measurable subsets $A, B \subset X$. Let $g_{n} \in F_{n+1} \backslash F_{n}$. It suffices to show that a subsequence of $\left(g_{n}\right)_{n=1}^{\infty}$ is mixing for $T$. We write $g_{n}=f_{n} \phi_{n}\left(h_{n}\right)$ for some $f_{n} \in \widetilde{F}_{n} \cap C(G)$ and $h_{n} \in H_{n}$. Denote by $z: \mathbb{Z} \rightarrow C(G)$ the natural embedding $z(x):=(x, 0,0)$. We may assume that $f_{n} \in z\left(\mathbb{Z}_{+}\right)$for all $n$ (the case $f_{n} \in z\left(\mathbb{Z}_{-}\right)$is considered in a similar way). Let $H_{n}^{\prime}:=H_{n} \cap\left(H_{n}-h_{n}\right)$ and $F_{n}^{\prime}:=F_{n} \cap\left(f_{n}^{-1} F_{n}\right)$. Passing to a subsequence, if necessary, we also may assume without loss of generality that

$$
\frac{\left|H_{n}^{\prime}\right|}{\left|H_{n}\right|} \rightarrow \delta_{1} \quad \text { and } \quad \frac{\lambda\left(F_{n}^{\prime}\right)}{\lambda\left(F_{n}\right)} \rightarrow \delta_{2}
$$

for some $\delta_{1}, \delta_{2} \geqslant 0$. Partition $H_{n}$ into three subsets $H_{n}^{1}, H_{n}^{2}$ and $H_{n}^{3}$ as follows

$$
\begin{aligned}
& H_{n}^{1}:=\left\{h \in H_{n} \mid g_{n} F_{n} c_{n+1}(h) \subset F_{n+1} \phi_{n+1}\left(h_{0}\right)\right\}, \\
& H_{n}^{2}:=\left\{h \in H_{n} \mid g_{n} F_{n} c_{n+1}(h) \subset F_{n+1}\right\}, \\
& H_{n}^{3}:=H_{n} \backslash\left(H_{n}^{1} \sqcup H_{n}^{2}\right) .
\end{aligned}
$$

As before $h_{0}=(1,0) \in \mathbb{Z}^{2}$. Let $C_{n+1}^{i}:=\phi_{n+1}\left(H_{n}^{i}\right)$. It is clear that $\left|H_{n}^{3}\right| \leqslant$ $4(n+1)\left(2 r_{n}+1\right)$ and $\left|H_{n}^{2} \triangle H_{n}^{\prime}\right| \leqslant 2 r_{n}+1$. Since $\left|H_{n}\right|=\left(2 r_{n}+1\right)^{2}$, it follows that

$$
\frac{\left|H_{n}^{1}\right|}{\left|H_{n}\right|} \rightarrow 1-\delta_{1}, \quad \frac{\left|H_{n}^{2}\right|}{\left|H_{n}\right|} \rightarrow \delta_{1}, \quad \frac{\left|H_{n}^{3}\right|}{\left|H_{n}\right|} \rightarrow 0 .
$$

Take two $\xi_{n}$-measurable subsets $A, B \subset F_{n}$. Since

we have

$$
\mu\left(\left[A C_{n+1}^{3}\right]_{n+1}\right)=\frac{\left|C_{n+1}^{3}\right|}{\left|C_{n+1}\right|} \mu\left([A]_{n}\right) \leqslant \frac{1}{2 r_{n}+1} \rightarrow 0,
$$

$$
\left|\mu\left(T_{g_{n}}\left[A C_{n+1}^{3}\right]_{n+1} \cap[B]_{n}\right)-\mu\left(\left[A C_{n+1}^{3}\right]_{n+1}\right) \mu\left([B]_{n}\right)\right| \rightarrow 0,
$$

so $\left[F_{n} C_{n+1}^{3}\right]_{n+1}$ is negligible. It suffices to show mixing separately on each of the remaining subsets $\left[F_{n} C_{n+1}^{1}\right]_{n+1}$ and $\left[F_{n} C_{n+1}^{2}\right]_{n+1}$. 
First, we note that $\phi_{n+1}\left(h_{0}\right)^{-1} g_{n} F_{n} C_{n+1}^{1} \subset F_{n+1}$. Thus, by (1.12),

$$
T_{g_{n}}\left[A C_{n+1}^{1}\right]_{n+1}=T_{\phi_{n+1}\left(h_{0}\right)}\left[\phi_{n+1}\left(h_{0}\right)^{-1} g_{n} A C_{n+1}^{1}\right]_{n+1} .
$$

By Lemma 2.6 (with $C_{n+1}^{*}:=\phi_{n+1}\left(h_{0}\right)^{-1} \phi_{n}\left(h_{n}\right) C_{n+1}^{1}$ and $A^{*}:=f_{n} A$ ) we obtain that

$$
\left|\mu\left(T_{g_{n}}\left[A C_{n+1}^{1}\right]_{n+1} \cap[B]_{n}\right)-\mu\left(\left[A C_{n+1}^{1}\right]_{n+1}\right) \mu\left([B]_{n}\right)\right| \rightarrow 0 .
$$

It remains to consider the second case involving $C_{n+1}^{2}$. If $\delta_{1}=0$, then obviously

$$
\mu\left(\left[A C_{n+1}^{2}\right]_{n+1}\right) \rightarrow 0 \text {. }
$$

Suppose now that $\delta_{1}>0$. Partition $A$ into three subsets $A_{1}, A_{2}$ and $A_{3}$ in the following way: $A_{1}:=A \cap f_{n}^{-1} F_{n}, A_{2}:=A \cap f_{n}^{-1} F_{n} \phi_{n}\left(h_{0}\right)$ and $A_{3}:=A \backslash\left(A_{1} \sqcup A_{2}\right)$. In other words, $f_{n} A_{1} \subset F_{n}, f_{n} A_{2} \subset F_{n} \phi_{n}\left(h_{0}\right), f_{n} A_{3} \cap\left(F_{n} \sqcup F_{n} \phi_{n}\left(h_{0}\right)\right)=\varnothing$.

Note that

$$
\mu\left(\left[A_{3} C_{n+1}^{2}\right]_{n+1}\right) \leqslant \mu\left(\left[A_{3}\right]_{n}\right) \leqslant \frac{2 n+1}{2 r_{n}+1} \rightarrow 0 .
$$

For $A_{1}$ and $A_{2}$ we argue as in the proof of Lemma 2.6. Set $F_{n}^{\circ}:=\left\{f \in F_{n} \mid\right.$ $\left.f S_{n} S_{n}^{-1} \subset F_{n}\right\}, A_{1}^{\circ}:=A_{1} \cap F_{n}^{\circ}, B^{\circ}:=B \cap F_{n}^{\circ}$. We have

$$
\begin{aligned}
\mu\left(T_{g_{n}}\left[A_{1} C_{n+1}^{2}\right]_{n+1} \cap[B]_{n}\right)=\sum_{h \in H_{n}^{\prime}} \mu\left(\left[\phi_{n}\left(h_{n}\right) f_{n} A_{1}^{\circ} c_{n+1}(h)\right]_{n+1} \cap\left[B^{\circ}\right]_{n}\right)+\bar{o}(1) \\
=\sum_{h \in H_{n}^{\prime}} \mu\left(\left[\left(f_{n} A_{1}^{\circ} s_{n}(h) s_{n}\left(h_{n}+h\right)^{-1} \cap B^{\circ}\right) c_{n+1}(h)\right]_{n+1}\right)+\bar{o}(1) \\
=\frac{1}{\left|H_{n}\right|} \sum_{h \in H_{n}^{\prime}} \mu\left(\left[f_{n} A_{1}^{\circ} s_{n}(h) s_{n}\left(h_{n}+h\right)^{-1} \cap B^{\circ}\right]_{n}\right)+\bar{o}(1) \\
=\frac{\delta_{1}}{\left|H_{n}^{\prime}\right|} \sum_{h \in H_{n}^{\prime}} \lambda_{F_{n}}\left(f_{n} A_{1}^{\circ} s_{n}(h) \cap B^{\circ} s_{n}\left(h_{n}+h\right)_{n}\right)+\bar{o}(1) \\
=\delta_{1} \int_{S_{n} \times S_{n}} f_{A_{1} f_{n}, B} d \nu_{n}+\bar{o}(1),
\end{aligned}
$$

where $\nu_{n}:=\operatorname{dist}_{h \in H_{n}^{\prime}}\left(s_{n}(h), s_{n}\left(h_{n}+h\right)\right)$ and $f_{A_{1} f_{n}, B}(x, y)=\lambda_{F_{n}}\left(A_{1} f_{n} x \cap B y\right)$. Write $h_{n}=\left(t_{n}, 0\right)$. Since $\frac{2 r_{n}-t_{n}+1}{2 r_{n}+1}=\frac{\left|H_{n}^{\prime}\right|}{\left|H_{n}\right|} \rightarrow \delta_{1}>0$ and

$$
\nu_{n}=\frac{1}{2 r_{n}-1} \sum_{i=-r_{n}}^{r_{n}} \operatorname{dist}_{-r_{n} \leqslant t \leqslant r_{n}-t_{n}}\left(s_{n}(t, i), s_{n}\left(t+t_{n}, i\right)\right),
$$

it follows from (2.6) and (2.5) that

$$
\mu\left(T_{g_{n}}\left[A_{1} C_{n+1}^{2}\right]_{n+1} \cap[B]_{n}\right)=\frac{\delta_{1}}{\lambda\left(S_{n}\right)^{2}} \int_{S_{n} \times S_{n}} f_{A_{1} f_{n}, B} d \lambda d \lambda+\bar{o}(1) .
$$

Now take $A:=A^{*} C_{n}^{*}$ and $B:=B^{*} C_{n}$ for some $\xi_{n-1}$-measurable subsets $A^{*}, B^{*} \subset$ $F_{n-1}$. Let $C_{n}^{\prime}:=C_{n} \cap F_{n}^{\prime}$. It follows that $\frac{\left|C_{n}^{\prime}\right|}{\left|C_{n}\right|} \rightarrow \delta_{2}$ and $\mu\left(\left[A_{1}\right]_{n} \triangle\left[A^{*} C_{n}\right]_{n}\right)=\bar{o}(1)$. Hence $\mu\left(\left[A_{1}\right]_{n}\right)=\delta_{2} \mu\left(\left[A^{*}\right]_{n-1}\right)+\bar{o}(1)$. Arguing as in the proof of Lemma 2.6] we obtain that

$$
\mu\left(T_{g_{n}}\left[A^{*} C_{n}^{\prime} C_{n+1}^{2}\right]_{n+1} \cap\left[B^{*}\right]_{n-1}\right)=\delta_{2} \mu\left(\left[A^{*}\right]_{n-1}\right) \mu\left(\left[B^{*}\right]_{n-1}\right)+\bar{o}(1) .
$$

Therefore

$$
\left|\mu\left(T_{g_{n}}\left[A_{1} C_{n+1}^{2}\right]_{n+1} \cap[B]_{n}\right)-\mu\left(\left[A_{1} C_{n+1}^{2}\right]_{n+1}\right) \mu\left([B]_{n}\right)\right| \rightarrow 0 .
$$


Since $T_{g_{n}}\left[A_{2}\right]_{n}=T_{\phi_{n}\left(h_{n}+h_{0}\right)}\left[\phi_{n}\left(h_{0}\right)^{-1} f_{n} A_{2}\right]$ with $\phi_{n}\left(h_{0}\right)^{-1} f_{n} A_{2} \subset F_{n}$, a similar reasoning yields

$$
\left|\mu\left(T_{g_{n}}\left[A_{2} C_{n+1}^{2}\right]_{n+1} \cap[B]_{n}\right)-\mu\left(\left[A_{2} C_{n+1}^{2}\right]_{n+1}\right) \mu\left([B]_{n}\right)\right| \rightarrow 0 .
$$

Since

$$
\left[A^{*}\right]_{n-1}=\left[A^{*} C_{n} C_{n+1}^{1}\right]_{n+1} \sqcup \bigsqcup_{i=1}^{3}\left[A_{i} C_{n+1}^{2}\right]_{n+1} \sqcup\left[A^{*} C_{n} C_{n+1}^{3}\right]_{n+1},
$$

it follows from (2.10) -2.15$)$ that

$$
\lim _{n \rightarrow \infty} \sup _{A^{*}, B^{*} \in \sigma\left(\xi_{n-1}\right)}\left|\mu\left(T_{g_{n}}\left[A^{*}\right]_{n-1} \cap\left[B^{*}\right]_{n-1}\right)-\mu\left(\left[A^{*}\right]_{n-1}\right) \mu\left(\left[B^{*}\right]_{n-1}\right)\right|=0 .
$$

Since $\xi_{n}$-measurable cylinders generate the entire $\sigma$-algebra $\mathfrak{B}$ as $n \rightarrow \infty$, it follows that $\left(g_{n}\right)_{n=1}^{\infty}$ is a mixing sequence for $T$, as desired.

Proposition 2.9. The transformation $T_{(1,0,0)}$ is 2-fold simple and $C\left(T_{(1,0,0)}\right)=$ $\left\{T_{g} \mid g \in G\right\}$.

Proof. Take an ergodic joining $\nu \in J_{2}^{e}\left(T_{(1,0,0)}\right)$. Let $K_{n}:=\left[-\frac{a_{n}}{n^{2}}, \frac{a_{n}}{n^{2}}\right]_{\mathbb{Z}}, J_{n}:=$ $\left[-\frac{r_{n}}{n^{2}}, \frac{r_{n}}{n^{2}}\right]_{\mathbb{Z}}$ and $\Phi_{n}:=K_{n}+2 \widetilde{a}_{n} J_{n}$. We claim that $\nu$-a.e. point $(x, y) \in X \times X$ is generic for $T_{(1,0,0)} \times T_{(1,0,0)}$, i.e. for all cylinders $A, B \subset \bigcup_{n=1}^{\infty} \sigma\left(\xi_{n}\right)$ we have

$$
\nu(A \times B)=\lim _{n \rightarrow \infty} \frac{1}{\left|\Phi_{n}\right|} \sum_{i \in \Phi_{n}} \chi_{A}\left(T_{(i, 0,0)} x\right) \chi_{B}\left(T_{(i, 0,0)} y\right) .
$$

To see this, we first note that $\left(\Phi_{n}\right)_{n=1}^{\infty}$ is a Følner sequence in $\mathbb{Z}$. Since

$$
\frac{a_{n}}{n^{2}}+\frac{2 \widetilde{a}_{n} r_{n}}{n^{2}}<\frac{\widetilde{a}_{n}\left(2 r_{n}+1\right)}{n^{2}}<\frac{2 a_{n+1}}{(n+1)^{2}},
$$

it follows that $\Phi_{n} \subset K_{n+1}+K_{n+1}$ and hence $\bigcup_{m=1}^{n} \Phi_{m} \subset K_{n+1}+K_{n+1}$. This implies that $\left|\Phi_{n+1}+\bigcup_{m \leqslant n} \Phi_{m}\right| \leqslant 3\left|\Phi_{n+1}\right|$ for every $n \in \mathbb{N}$, i.e. Shulman's condition [Li] is satisfied for $\left(\Phi_{n}\right)_{n=1}^{\infty}$. By [Li], the pointwise ergodic theorem holds along $\left(\Phi_{n}\right)_{n=1}^{\infty}$ for any ergodic transformation. Since $T \times T$ is $\nu$-ergodic, 2.16) holds for $\nu$-a.a. $(x, y) \in X \times X$ and for every pair of cylinders $A, B \subset X$ from $\bigcup_{n=1}^{\infty} \sigma\left(\xi_{n}\right)$.

Fix a generic point $(x, y) \in X \times X$. Since $x, y \in X_{n}$ for all sufficiently large $n$ and we have the following expansion

$$
\begin{aligned}
& x=\left(f_{n}, c_{n+1}\left(h_{n}\right), c_{n+2}\left(h_{n+1}\right), \ldots\right), \\
& y=\left(f_{n}^{\prime}, c_{n+1}\left(h_{n}^{\prime}\right), c_{n+2}\left(h_{n+1}^{\prime}\right), \ldots\right)
\end{aligned}
$$

with $f_{n}, f_{n}^{\prime} \in F_{n}, h_{i}, h_{i}^{\prime} \in H_{i}, i \geqslant n$. We let $H_{n}^{-}:=\left[-\left(1-\frac{1}{n^{2}}\right) r_{n},\left(1-\frac{1}{n^{2}}\right) r_{n}\right]_{\mathbb{Z}}^{2} \subset$ $H_{n}$. Since the marginals of $\nu$ both equal to $\mu$, we may assume without loss of generality that $h_{n}, h_{n}^{\prime} \in H_{n}^{-}$. Indeed,

$$
\mu\left(\left\{x=\left(f_{n}, c_{n+1}\left(h_{n}\right), c_{n+2}\left(h_{n+1}\right), \ldots\right) \in X_{n} \mid h_{n} \notin H_{i}^{-}\right\}\right)<\frac{2}{i^{2}},
$$

and hence by Borel-Cantelli lemma for $\mu$-a.e. $x \in X_{n}$ and all but finitely many $i$ we have $h_{i} \in H_{i}^{-}$. Then we may replace $x=\left(f_{n}, c_{n+1}\left(h_{n}\right), c_{n+2}\left(h_{n+1}\right), \ldots\right) \in X_{n}$ with $x=\left(f_{n} c_{n+1}\left(h_{n}\right) \cdots c_{m}\left(h_{m-1}\right), c_{m+1}\left(h_{m}\right), \ldots\right) \in X_{m}$ for some $m>n$ if necessary. Similarly, $h_{n}^{\prime} \in H_{n}^{-}$.

This implies, in turn, that 


$$
f_{n+1}=f_{n} c_{n+1}\left(h_{n}\right) \in \widetilde{F}_{n} \phi_{n}\left(H_{n}^{-}\right) \subset\left[-c_{n}, c_{n}\right]_{\mathbb{Z}} \times\left[-c_{n}, c_{n}\right]_{\mathbb{R}} \times \mathbb{Z}_{2},
$$

where $c_{n}=\widetilde{a}_{n}\left(1+2 r_{n}\left(1-\frac{1}{n^{2}}\right)\right)$, and, similarly, $f_{n+1}^{\prime} \in\left[-c_{n}, c_{n}\right]_{\mathbb{Z}} \times\left[-c_{n}, c_{n}\right]_{\mathbb{R}} \times \mathbb{Z}_{2}$.

Given $g \in \Phi_{n}$, there are some uniquely determined $k \in K_{n}$ and $j \in J_{n}$ such that $g=k+2 \widetilde{a}_{n} j$, i.e. $(g, 0,0)=(k, 0,0) \phi_{n}(j, 0)$. Moreover, $(j, 0)+h_{n} \in H_{n}$ since $h_{n} \in H_{n}^{-}$. It also follows from (2.17) that

$$
(k, 0,0) f_{n} S_{n} S_{n}^{ \pm 1} \subset F_{n} .
$$

Take $g \in \Phi_{n}$ and calculate $T_{(g, 0,0)} x$.

$$
\begin{aligned}
x=\left(f_{n}, c_{n+1}\left(h_{n}\right), \ldots\right) & =\left(f_{n} c_{n+1}\left(h_{n}\right), \ldots\right)=\left(f_{n} s_{n}\left(h_{n}\right) \phi_{n}\left(h_{n}\right), \ldots\right) . \\
(g, 0,0) f_{n} s_{n}\left(h_{n}\right) \phi_{n}\left(h_{n}\right) & =(k, 0,0) \phi_{n}(j, 0) f_{n} s_{n}\left(h_{n}\right) \phi_{n}\left(h_{n}\right) \\
& =(k, 0,0) f_{n} s_{n}\left(h_{n}\right) \phi_{n}\left((j, 0)+h_{n}\right) \\
& =(k, 0,0) f_{n} s_{n}\left(h_{n}\right) s_{n}\left((j, 0)+h_{n}\right)^{-1} c_{n+1}\left((j, 0)+h_{n}\right) \\
& =d c_{n+1}\left((j, 0)+h_{n}\right),
\end{aligned}
$$

where $d:=(k, 0,0) f_{n} s_{n}\left(h_{n}\right) s_{n}\left((j, 0)+h_{n}\right)^{-1} \in F_{n}$ by (2.18). This means that $T_{(g, 0,0)} x=(d, \ldots) \in X_{n}$. Similarly,

$$
(g, 0,0) f_{n}^{\prime}, s_{n}\left(h_{n}^{\prime}\right) \phi_{n}\left(h_{n}^{\prime}\right)=d^{\prime} c_{n+1}\left((j, 0)+h_{n}^{\prime}\right)
$$

with $d^{\prime}:=(b, 0,0) f_{n}^{\prime} s_{n}\left(h_{n}^{\prime}\right) s_{n}\left((t, 0)+h_{n}^{\prime}\right)^{-1} \in F_{n}$.

Now take any $\xi_{n-2}$-measurable subsets $A^{*}, B^{*} \subset F_{n-2}$ and set $A:=A^{*} C_{n-1} C_{n}$, $B:=B^{*} C_{n-1} C_{n}$.

$$
\begin{aligned}
\nu\left(\left[A^{*}\right]_{n-2}\right. & \left.\times\left[B^{*}\right]_{n-2}\right)=\nu\left([A]_{n} \times[B]_{n}\right) \\
& =\lim _{n \rightarrow \infty} \frac{\left|\left\{g \in \Phi_{n} \mid T_{(g, 0,0)} x \in[A]_{n}, T_{(g, 0,0)} y \in[B]_{n}\right\}\right|}{\left|\Phi_{n}\right|} \\
& =\lim _{n \rightarrow \infty} \frac{\left|\left\{g \in \Phi_{n} \mid d \in A, d^{\prime} \in B\right\}\right|}{\left|\Phi_{n}\right|} \\
& =\lim _{n \rightarrow \infty} \frac{1}{\left|K_{n}\right|} \sum_{k \in K_{n}} \frac{\left|\left\{j \in J_{n} \mid d \in A, d^{\prime} \in B\right\}\right|}{\left|J_{n}\right|} \\
& =\lim _{n \rightarrow \infty} \frac{1}{\left|K_{n}\right|} \sum_{k \in K_{n}} \zeta_{n}\left(A^{-1}(k, 0,0) f_{n} s_{n}\left(h_{n}\right) \times B^{-1}(k, 0,0) f_{n}^{\prime} s_{n}\left(h_{n}^{\prime}\right)\right),
\end{aligned}
$$

where $\zeta_{n}:=\operatorname{dist}_{j \in J_{n}}\left(s_{n}\left((j, 0)+h_{n}\right), s_{n}\left((j, 0)+h_{n}^{\prime}\right)\right)$. We consider separately two cases.

First case. Suppose first that $h_{n} \neq h_{n}^{\prime}$ for infinitely many, say bad $n$. Since $\left|J_{n}\right| \geqslant \frac{r_{n}}{n^{2}}$ it follows from (2.6) that $\left\|\zeta_{n}-\kappa_{D_{n}} \times \kappa_{D_{n}}\right\|<\frac{1}{n}$. Moreover, it follows from (2.18) and (2.5) (we need (2.5) for $A^{-1}(k, 0,0) f_{n} s_{n}(h)$ ) that

$$
\kappa_{D_{n}}\left(A^{-1}(k, 0,0) f_{n} s_{n}(h)\right)=\lambda_{S_{n}}\left(A^{-1}(k, 0,0) f_{n} s_{n}(h)\right)+\bar{o}(1) .
$$

Hence

$$
\begin{aligned}
& \frac{1}{\left|K_{n}\right|} \sum_{k \in K_{n}} \zeta_{n}\left(A^{-1}(k, 0,0) f_{n} s_{n}\left(h_{n}\right) \times B^{-1}(k, 0,0) f_{n}^{\prime} s_{n}\left(h_{n}^{\prime}\right)\right) \\
& =\frac{1}{\left|K_{n}\right|} \sum_{k \in K_{n}} \kappa_{D_{n}}\left(A^{-1}(k, 0,0) f_{n} s_{n}\left(h_{n}\right)\right) \kappa_{D_{n}}\left(B^{-1}(k, 0,0) f_{n}^{\prime} s_{n}\left(h_{n}^{\prime}\right)\right)+\bar{o}(1)
\end{aligned}
$$




$$
=\frac{1}{\left|K_{n}\right|} \sum_{k \in K_{n}} \lambda_{S_{n}}\left(A^{-1}(k, 0,0) f_{n} s_{n}\left(h_{n}\right)\right) \lambda_{S_{n}}\left(B^{-1}(k, 0,0) f_{n}^{\prime} s_{n}\left(h_{n}^{\prime}\right)\right)+\bar{o}(1)
$$

Now we derive from Lemma 2.3(ii) that

$$
\begin{aligned}
\lambda_{S_{n}}\left(A^{-1}(k, 0,0) f_{n} s_{n}\left(h_{n}\right)\right) & =\frac{\lambda\left(A^{-1}(k, 0,0) f_{n} s_{n}\left(h_{n}\right) \cap S_{n}\right)}{\lambda\left(S_{n}\right)} \\
& =\frac{\lambda\left(A \cap(k, 0,0) f_{n} s_{n}\left(h_{n}\right) S_{n}\right)}{\lambda\left(S_{n}\right)}=\lambda_{F_{n-2}}\left(A^{*}\right)+\bar{o}(1)
\end{aligned}
$$

and, in a similar way, $\lambda_{S_{n}}\left(B^{-1}(b, 0,0) f_{n}^{\prime} s_{n}\left(h_{n}^{\prime}\right)\right)=\lambda_{F_{n-2}}\left(B^{*}\right)+\bar{o}(1)$. Hence

$\nu\left(\left[A^{*}\right]_{n-2} \times\left[B^{*}\right]_{n-2}\right)=\lambda_{F_{n-2}}\left(A^{*}\right) \lambda_{F_{n-2}}\left(B^{*}\right)+\bar{o}(1)=\mu\left(\left[A^{*}\right]_{n-2}\right) \mu\left(\left[B^{*}\right]_{n-2}\right)+\bar{o}(1)$

for all bad $n$ and all $\xi_{n-2}$-measurable subsets $A^{*}, B^{*} \subset F_{n-2}$. Since any measurable set can be approximated by $\left[A^{*}\right]_{n-2}$, it follows that in this case $\nu=\mu \times \mu$.

Second case. Now we consider the case where $h_{n}=h_{n}^{\prime}$ for all $n$ greater than some $N$. Then it is easy to see that $y=T_{k} x$, where $k=f_{N}^{\prime} f_{N}^{-1} \in G$ and then it follows immediately that $(x, y)$ is generic for the off-diagonal joining $\mu_{T_{k}}$ :

$$
\begin{aligned}
& \nu\left([A]_{n} \times[B]_{n}\right)=\lim _{n \rightarrow \infty} \frac{1}{\left|\Phi_{n}\right|} \sum_{i \in \Phi_{n}} \chi_{[A]_{n}}\left(T_{(i, 0,0)} x\right) \chi_{[B]_{n}}\left(T_{(i, 0,0)} T_{k} x\right)= \\
= & \lim _{n \rightarrow \infty} \frac{1}{\left|\Phi_{n}\right|} \sum_{i \in \Phi_{n}} \chi_{[A]_{n} \cap T_{k}^{-1}[B]_{n}}\left(T_{(i, 0,0)} x\right)=\mu\left([A]_{n} \cap T_{k}^{-1}[B]_{n}\right)=\mu_{T_{k}}\left([A]_{n} \times[B]_{n}\right)
\end{aligned}
$$

for all $A, B \in \sigma\left(\xi_{n}\right)$, since $\nu$ projects onto $\mu$. Since each measurable set can be approximated by cylinder sets, we deduce that in this case $\nu=\mu_{T_{k}}$ with $k \in G$.

Proof of Theorem 2.2. follows now from Veech's theorem, Propositions 2.8, 2.9 and the fact that $\mathfrak{F}_{G_{a}}$ and $\mathfrak{F}_{G_{b}}$ are isomorphic if and only if $G_{a}$ and $G_{b}$ are conjugate in $G$ dJR, Corollary 3.3]. It is clear, that $G_{b}=h G_{a} h^{-1}$ with $h=\left(0, \frac{a+b}{2}, 1\right)$.

Notice that with some additional conditions on $s_{n}$ in Lemma 2.1 (cf. [Da3, Lemma 2.3]) one can show that $T_{(1,0,0)}$ is actually mixing of all orders.

\section{Concluding Remarks}

If we replace $G=\mathbb{Z} \times\left(\mathbb{R} \rtimes \mathbb{Z}_{2}\right)$ with $\Gamma:=\mathbb{R} \times\left(\mathbb{R} \rtimes \mathbb{Z}_{2}\right)$ and apply the same construction (with obvious minor changes) we obtain a probability preserving $\Gamma$ action $R$ such that the flow $\left(R_{(t, 0,0)}\right)_{t \in \mathbb{R}}$ is 2 -fold simple mixing and its centralizer coincides with the entire $\Gamma$-action. This gives an example of 2 -fold simple mixing flow with uncountably many prime factors. By $[\mathrm{Ry}$, each 2-fold simple flow is simple. For the definitions of higher order simplicity we refer to dJR. Moreover, since $\mathbb{Z} \subset \mathbb{R}$ is a closed cocompact subgroup, the corresponding $\mathbb{Z}$-subaction is also 2-fold simple and $C\left(R_{(1,0,0)}\right)=\left\{R_{g} \mid g \in \Gamma\right\}$ by [dJR, Theorem 6.1]. Thus we get examples of two nonisomorphic 2 -fold simple transformations with uncountably many prime factors: $R_{(1,0,0)}$ is embeddable into a flow while $T_{(1,0,0)}$ is not.

\section{REFERENCES}

[Da1] A. I. Danilenko, $(C, F)$-actions in ergodic theory, in Geometry and Dynamics of Groups and Spaces, Progr. Math. 265 (2008), 325-351.

[Da2] A. I. Danilenko, Mixing actions of Heisenberg group, Ergod. Th. \& Dynam. Sys. (to appear), available on CJO2013. doi:10.1017/etds.2012.169. 
[Da3] A. I. Danilenko, Mixing rank-one actions for infinite sums of finite groups, Isr. J. Math., 156 (2006), 341-358

[DdJ] A. I. Danilenko and A. del Junco, Cut-and-stack simple weakly mixing map with countably many prime factors, Proc. Amer. Math. Soc. 136 (2008), 2463-2472.

[dJ] A. del Junco, A simple map with no prime factors, Israel J. Math. 104 (1998), 301-320.

[dJR] A. del Junco and D. Rudolph, On ergodic actions whose self-joinings are graphs, Ergod. Th. \& Dynam. Sys. 7 (1987), 531-557.

[Fe] S. Ferenczi, Systemes de rang un gauche, Ann. Inst. H. Poincare. Probab. Statist. 21 (1985), $177-186$.

[GlW] E. Glasner, B. Weiss, A simple weakly mixing transformation with nonunique prime factors, Amer. J. Math. 116 (1994), 361-375.

[Li] E. Lindenstrauss, Pointwise theorems for amenable groups, Invent. Math. 146 (2001), 259295.

[Ry] V. V. Ryzhykov, Around simple dynamical systems. Induced joinings and multiple mixing, J. Dynam. Control Systems 3 (1997), 111-127.

[So] A. Sokhet, Les actions approximativement transitives dans la théory ergodique, Thèse de doctorat, Université Paris VII, 1997.

[Ve] W. A. Veech, A criterion for a process to be prime, Monatsh. Math. 94 (1982), 335-341.

Institute for Low Temperature Physics \& Engineering of the National Academy of Sciences of Ukraine, 47 Lenin Ave., Kharkiv, 61103, UKRAINE

E-mail address: alexandre.danilenko@gmail.com

E-mail address: solomko.anton@gmail.com 